\title{
A HYDROGEN SEPARATION MODULE BASED ON WAFER-SCALE MICROMACHINED PALLADIUM-SILVER ALLOY MEMBRANES
}

\author{
H.D.Tong ${ }^{a}$, F.C.Gielens ${ }^{\text {b }}$, H.T.Hoang ${ }^{\text {a }}$, J.W.Berenschot ${ }^{\text {a }}$, M.J.De Boer ${ }^{\text {a }}$ \\ J.G.E.Gardeniers ${ }^{a}$, H.V.Jansen ${ }^{a}$, W.Nijdam ${ }^{a_{1}}$ C.J.M. van Rijn ${ }^{c}$, M.C.Elwenspoek ${ }^{a}$ \\ ${ }^{a} \mathrm{MESA}+$ research institute, University of Twente, The Netherlands \\ ${ }^{b}$ Department of Chemical Engineering and Chemistry, Eindhoven University of Technology, The Netherlands. ${ }^{c}$ \\ Aquamarijn Micro Filtration B.V., The Netherlands \\ Tel: (00 31) 53489 2805; Fax: (00 31) 53489 3343; Email: T.Hien@el.utwente.nl
}

\begin{abstract}
A thin but strong and defect free palladium-silver (Pd-Ag) alloy membrane is fabricated with a sequence of well-known thin film and micromachining techniques. A microfabrication process also creates a robust wafer-scale membrane module, which is easy to be integrated into a membrane holder to have gastight connections to the outer world. The microfabricated membranes have been tested to determine the mechanical strength, 'hydrogen permeability and hydrogen selectivity. The membranes have high mechanical strength and can withstand pressures up to 3 bars at room temperature. High flow rates of up to $3.6 \mathrm{~mol} \mathrm{H}_{2}$ $/ \mathrm{m}^{2}$.s have been measured with a minimal selectivity of 1500 for $\mathrm{H}_{2} / \mathrm{He}$. The membranes survived operation at $450{ }^{\circ} \mathrm{C}$, which is a temperature relevant for practical application in industry, for more than 1000 hours.
\end{abstract}

\section{INTRODUCTION}

Hydrogen has been extensively used in many industrial sectors from petroleum refining, petrochemical to material processing, and in new energy-related applications such as clean fuel for fuel cells and vehicles [1-3]. Furthermore, the depletion of crude oil, natural gas and fossil fuel in combination with the stricter rules on environmental regulations have made hydrogen to be seriously considered as one of the alternative clean energy carriers in future [2-3].

Although the most abundant element in the universe, on earth hydrogen is mostly found chemically bonded. Chemical reactions are therefore necessary to break apart those bonds and free the hydrogen; in doing so a multicomponent gas stream of hydrogen and several other gases is formed. To have hydrogen with high purity for the end-uses, the gas stream is then separated by several separation techniques like a pressure swing adsorption or using of porous and nonporous membranes etc [2]. In particular, separation and purification of hydrogen by means of dense metallic membranes are of great importance and interest, as the technology is able to provide very high selectivity for hydrogen [4-6]. In addition, until diffusion is a limiting step in the hydrogen transport [4], the high hydrogen flux through the membrane can be achieved by using thin membranes because the permeation rate is inversely proportional to the membrane thickness. Moreover, the metallic membranes can also be used in membrane reactions involving hydrogenation or dehydrogenation [4-6].

Among many possible hydrogen membrane materials, Pd and its alloys are the most widely studied due to their high hydrogen permeability, chemical compatibility with many hydrocarbon containing gas streams, infinite hydrogen selectivity, and good mechanical stability [4-6]. And normally, $\mathrm{Pd}$ is alloyed with some elements like $\mathrm{Ag}, \mathrm{Au}, \mathrm{Cu}$, $\mathrm{Ni}$ etc. to overcome $\mathrm{a}^{2}$ well-known problem of hydrogen embrittlement [5]. A comprehensive review of Pd-based membranes has been recently provided by Shu et al. [4], Hughes [5] and Dittmeyer et al. [6].

The existing Pd-based membranes can be classified into two types, according to the structure of membrane:(1) self-supported dense metallic membranes, and (2) composite type structure composed of a thin selective layer deposited on porous supports.

Most commercially available Pd membranes are in the forms of tubes or foils (self-supported dense metallic membranes), fabricated by fine metallurgical technology. These membranes have an adequate mechanical strength and are available in the forms that are easily integrated into a separation set up. They are, however, relatively thick [7],
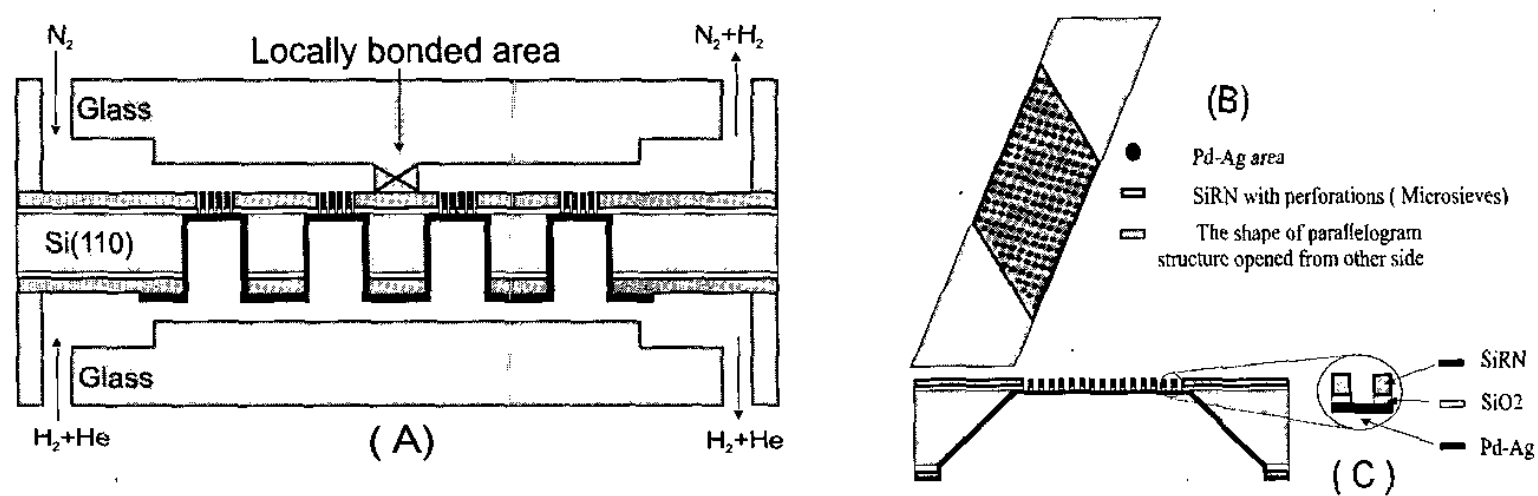

Figure 1. Separation membrane module: (A) Cross section; (B) Top view of membrane area creating by etching of one parallelogram-shaped structure in silicon wafer; (C) Side view of one parallelogram-shaped structure.

TRANSDUCERS '03

The 12th International Conference on Solid State Sensors, Actuators and Microsystems, Boston, June 8-12, 2003 


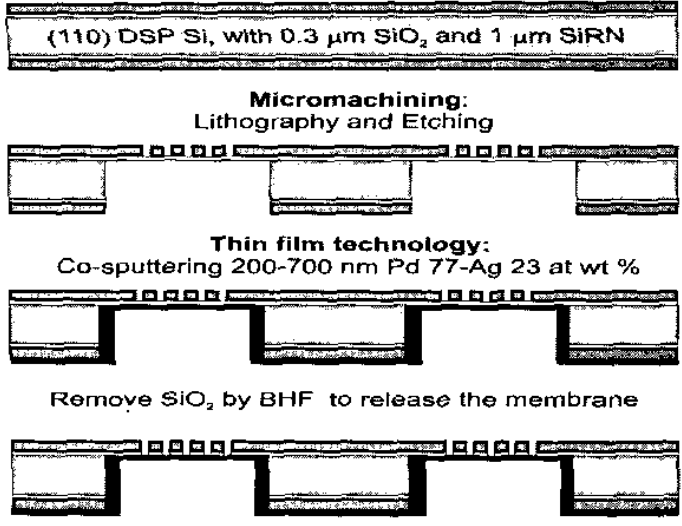

Figure 2. Process steps of the $\mathrm{Pd}-\mathrm{Ag}$ membrane.

consequently reducing the hydrogen flux and inhibiting their application for large-scale chemical production, apart from the investment cost of the precious metal.

In the last decade, several methods including sputtering, chemical vapour deposition (CVD), spray pyrolysis, electroless plating, electrodeposition and vacuum electrodeposition etc [4-6] have been developed to deposit thin Pd composite membranes onto porous substrates, which provide a mechanical strength. Generally, producing membranes as such is not too difficult, while the use of a thin Pd layer enhances the flux and reduces the membranes cost. To make submicron thick separation layers without pin-holes and cracks (defect free) is, however, very difficult $[4,6]$.

Recently, microfabrication techniques, originally developed for semiconductor technology and extended to micro-electromechanical systems, are increasingly used in different fields of chemistry and biotechnology [8-12]. By a combination of miniaturization with an integration of different components like sensors (temperature and flows), heaters, mixers, valves, pumps etc. into one system, microchemical devices and systems achieve many benefits and have capabilities exceeding those of conventional macroscopic system [8-12]. For instance, in addition to many already demonstrated chemical and biological analysis applications [11], microchemical systems are expected to have numerous advantages for chemical kinetics studies [10] or on-site toxic and hazardous chemical synthesis, and process development etc [12]. In all of the mentioned examples, microchemical devices and systems are advantageous in large part simply because they are smaller and more compact - a trend for process miniaturization [13].

Moreover, researchers from the field of process engineering have realized that the advantages of microstructured components are not only limited to simple miniaturization, and a number of highly promising applications involving moderate and, in some cases, even large quantities of matter and or/energy have begun to emerge in the rapidly growing field of process intensification [13]. In this trend, microfabrication technology is not simply utilized to make devices smaller, but rather to make them better - a trend for process intensification [13].

In recent years, considerable effort has been spent to utilize microfabrication technology as a novel approach for fabricating very thin, pin-hole free Pd composite membranes for hydrogen separation or hydrogenation/dehydrogenation reaction [12,14-15]. For example, a small size, microfabricated $\mathrm{Pd}$ membrane reported by Frank et al. [14] achieved high flux and high selectivity and is suitable for hydrogen purification in laboratory, where normally a small, high quality amount of hydrogen is required.

However, in industrial hydrogen separation, where a large amount of hydrogen has to be separated, microfabricated membranes may gain more benefit if the membrane is made to follow the second trend- the trend for process intensification. The current paper focuses on the microfabrication of a defect free, submicron thick $\mathrm{Pd}-\mathrm{Ag}$ membrane as a completely robust membrane module. Moreover, the membrane performance, was characterized with respect to mechanical strength, hydrogen flux and selectivity.

\section{MICROFABRICATION OF THE MEMBRANE MODULE}

A cross-section of the Pd-Ag membrane module and a membrane design are shown in fig. 1 (A). A Pd-Ag membrane is microfabricated on a silicon wafer, which is then sandwiched between two thick glass wafers.

\section{Microfabrication of Pd-Ag membrane on silicon wafer}

A microfabrication process for the Pd-Ag membrane on the silicon wafer, a key part of the membrane module, is presented in the following section.

The process steps of the Pd-Ag membrane on silicon wafer are shown in Fig.2. A 3 inch, double-side polished silicon substrate $(110)$ is coated with $0.3 \mu \mathrm{m}$ of wet-thermal silicon dioxide $\left(\mathrm{SiO}_{2}\right)$ and $1 \mu \mathrm{m}$ of low stress silicon rich silicon nitride (SiRN) by LPCVD [16]. Parallelogram-shaped structures of 650 by $2600 \mu \mathrm{m}$ (see fig.1 (B)) are aligned and imprinted on the backside of the wafer by standard photolithography, followed by a dry $\left(\mathrm{CHF}_{3}+\mathrm{O}_{2}\right)$ etching of the $\mathrm{SiRN}$ and wet etching of the oxide layer, using a buffered oxide etch (BHF). A design of the parallelogram-shaped structure, which can be etched into (110) silicon wafer can be found in [17].

The wafer is immersed in $25 \% \mathrm{KOH}$ solution at $75^{\circ} \mathrm{C}$ to etch the silicon until ca. $30 \mu \mathrm{m}$ is left. Afterwards, the lithography and dry etching of SiRN are carried out to create a microsieve pattern with circular perforation of $5 \mu \mathrm{m}$ [18] on the front side of the wafer. Subsequently, the silicon inside the slits is removed by $\mathrm{KOH}$ etching, which stops on the $\mathrm{SiO}_{2}$ layer.

At this stage, an alloy film of $\mathrm{Pd}-\mathrm{Ag}$ is deposited by simultaneously sputtering from pure targets of $\mathrm{Pd}$ and $\mathrm{Ag}$ on the $\mathrm{SiO}_{2}$ inside the etched structures, using $\mathrm{Ti}$ as adhesion layer. By controlling the power supply for each single target, the sputtered rates of $\mathrm{Pd}$ and $\mathrm{Ag}$ were controlled exactly, thus allowing controlling a composition as well as a thickness of a dual deposited layer. In our work, the alloy film of Pd-Ag $(77-23$ wt. \%) with a thickness of $700 \mathrm{~nm}$ has been realized.

Finally, the $\mathrm{SiO}_{2}$ and the $\mathrm{Ti}$ are removed in $\mathrm{BHF}$ 
4B1.4

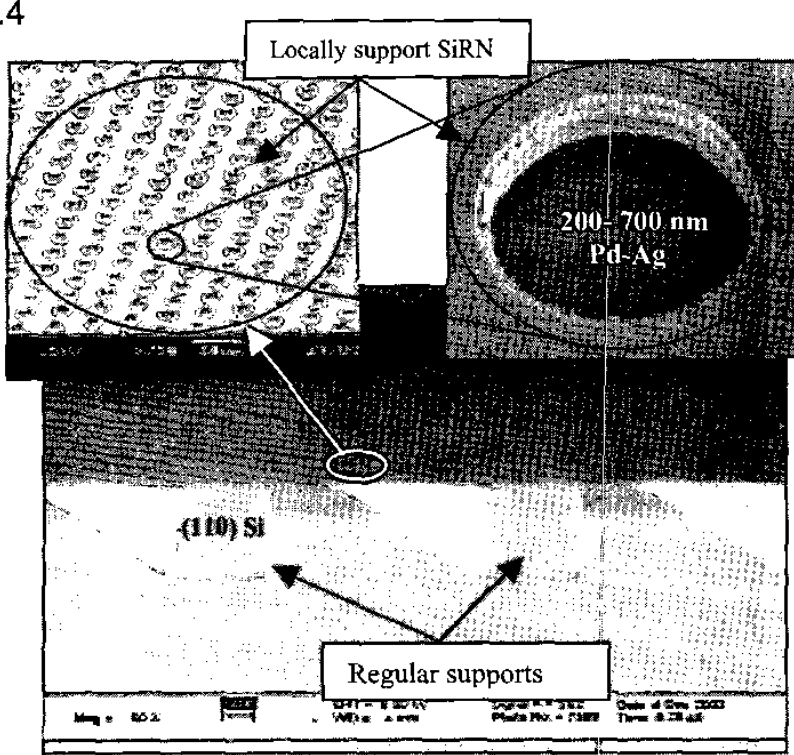

Figure3. SEM pictures of the microfabricated membrane: (110) supported structures, strong silicon nitride microsieves and $\mathrm{Pd}-\mathrm{Ag}$ membrane as seen through one Sieve.

through the opening of the SiRN microsieves to reveal the back surface of the Pd-Ag layer, thus forming $\mathrm{Pd}-\mathrm{Ag}$ membrane. Some SEM pictures of the microfabricated membrane are shown in fig.3.

\section{Fabrication of flow channels in glass}

As can be seen in fig.1, other parts of the membrane module are made of thick glass wafers, on which channels are created for gas flow. Moreover, a packaging with thick glass helps to improve the membrane mechanical robustness as well as protecting the membrane again harmful touches from outer environment. Powder blasting is used to create flow channels in a $5 \mathrm{~mm}$ thick glass wafer. The process steps of powder blasting to create a flow channel as well as a buffer zone on glass have been reported in detail in a previous paper [15]

\section{Assembly of the membrane module}

Finally, the silicon wafer is bonded between the two thick glass wafers by a four-electrode anodic bonding technique [15]. This process results in a tight seal between each glass wafer and the silicon wafer. Furthermore, the bonding process helps to create a robust membrane module, which is important for practical use (see fig.4). For example, this membrane module allows it to be integrated in a stainless steel membrane holder (see fig.5) to have connections to the outer world. In the membrane holder design, strong forces by screws are used to press membrane module and stainless steel plates tightly. An addition of flexible graphite rings is useful to make a gas-tight connection.

The method also allows the construction of a stack that consists of a large number of silicon wafers-a pilot scale, which is relevant for industrial applications where a high volume of hydrogen needs to be extracted from a gas mixtures.

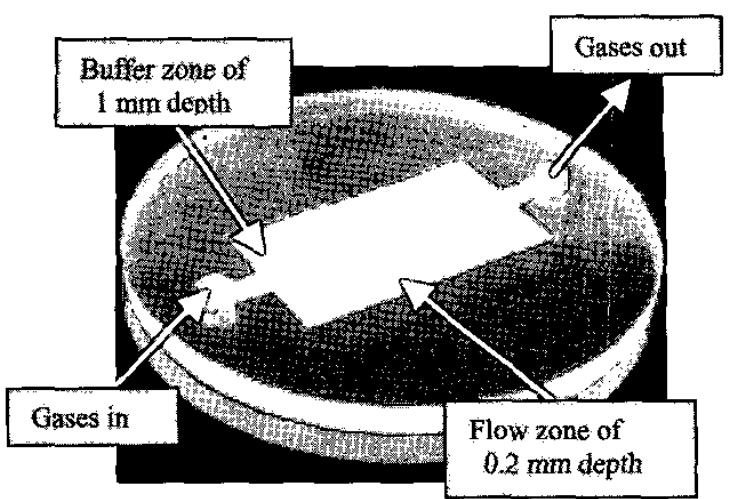

Figure 4. Strong and robust membrane module.

\section{RESULTS AND DISCUSSION}

\section{Mechanical strength of the membranes}

The mechanical strength of the microfabricated membranes is a vital aspect, as hydrogen flux is driven by a pressure gradient across the membrane. The rupture strength of the membrane at room temperature was measured in the set up that was described by Rijn et al. [18]. Preliminary tests showed that the Pd-Ag membranes did not break at a pressure difference of 3 bars between the two sides of the membrane. Although the membrane was rather thin $(700 \mathrm{~nm}$ thick), using different support structures such as a locally supported SiRN and regularly etched (110) structures (see fig.3) can be the reasons of achieving good membrane mechanical strength.

Although the rupture strength of the membrane has not measured at high temperature (operational membrane temperature) yet, the room temperature tests show that the microfabricated membrane is able to operate under desirable pressure gradient.

\section{Hydrogen permeation}

To determine hydrogen permeation and selectivity of the membranes, they were positioned in the stainless steel membrane holder and heated up to elevated temperatures. Next, the membrane permeability and selectivity for hydrogen were determined as a function of the hydrogen partial pressure $(0-0.9 \mathrm{bar})$, and temperature $\left(350-450^{\circ} \mathrm{C}\right)$. During experiments the retentate and permeate sides of the membrane were continuously flushed at atmospheric pressure, the retentate side with a mixture of hydrogen and helium, the permeate side with pure nitrogen. The flux and selectivity were determined by measuring the hydrogen and helium concentration in the nitrogen stream with a gas chromatograph, equipped with a Thermal Conductivity Detector (TCD). A detail of the measurement set-up has been previously reported by Gielens et al [19].

The hydrogen flow rates through the membrane versus the duration of the experiment are measured. At an average membrane temperature of $450^{\circ} \mathrm{C}$ and a hydrogen partial pressure of 0.83 bar at the feed side, a hydrogen flux of $3.6 \mathrm{~mol} \mathrm{H} \mathrm{H}_{2} / \mathrm{m}^{2} . \mathrm{s}$ was measured. The achieved flux is really high in comparison to the reported fluxes from 


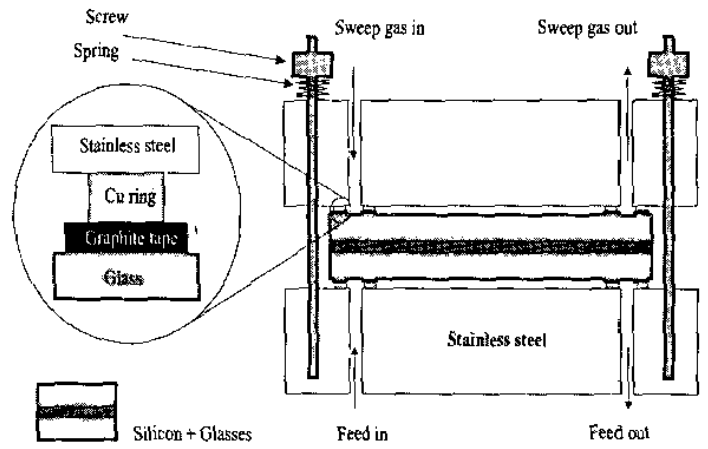

Figure 5. Wafer membrane module in a membrane holder.

literature [4-6, 14-15] and is due to the fact that; rather thin membranes have been used $(700 \mathrm{~nm})$. Such a membrane has a very low resistance to mass transfer in the gas phase, as virtually no support layer is present (see Fig.3).

Moreover, the dependence of the hydrogen flux on the hydrogen fraction in the feed was also investigated and shown in fig.6.As can be seen that the hydrogen flux almost depends linearly on the driving force $\left(\mathbf{p}_{\mathrm{H} 2 \text { retentate }}-\mathbf{p}_{\mathrm{H} 2}\right.$ permeate $)$. Since this is not according to Sieverts law, the fluxes through the thin membrane are not only limited by an atomic diffusion, other processes may contribute to the overall reaction rate $[4-6]$.

\section{Selectivity of the membranes}

From each sample taken, it is possible to detect a leak during the permeation experiment from the helium concentration. However, no helium was found in the permeate side during the experiments. Therefore, in order to calculate a minimum selectivity of hydrogen over helium, the detection limit of the $\mathrm{GC}$ for $\mathrm{He}$ is used as the maximum $\mathrm{He}$ concentration. A minimal separation factor of 1500 for hydrogen to helium (or equivalent to about 4000 for hydrogen to nitrogen) was calculated.

Although the highest selectivity of the membrane has not been able to be determined, the current minimal selectivity may be high enough to say that the microfabricated membrane is defect-free (pin-holes and cracks free). We believe that the membrane achieved such high selectivity because of several reasons. On the contrary with the conventional method, where the top separation layer has been deposited on rough and porous support substrates, leading to difficulties to obtain defect-free membranes, depositing a $\mathrm{Pd}-\mathrm{Ag}$ separation layer on a very smooth and clean surface of the sacrificial silicon oxide layer is a key advantage to get defect free membrane. Fabricating a membrane in a clean room environment is an advantage to obtain the thin defect free membrane.

\section{CONCLUSIONS}

The wafer-scale $\mathrm{Pd}-\mathrm{Ag}$ alloy membrane module was fabricated with microfabrication technology and tested. PdAg films were sputtered onto a smooth and clean surface of $\mathrm{SiO}_{2}$, creating defect free $\mathrm{Pd}-\mathrm{Ag}$ films. Moreover, local supports of the strong SiRN microsieves and regular supports of etched structures in (110) silicon wafer allow the fabrication of thin but strong Pd-Ag membranes. The units

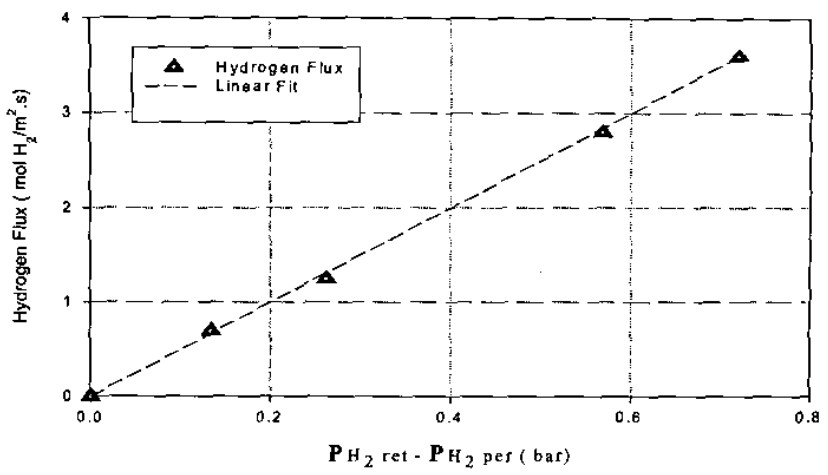

Figure 6. Dependence of hydrogen fluxes on hydrogen pressure in retentate side. The membrane was $700 \mathrm{~nm}$ thick of Pd-Ag. The temperature was kept at $450^{\circ} \mathrm{C}$, while average flow rates for periods of 100 hours were used for the graph.

are packaged in-between thick glass plates, using silicon-toglass wafer bonding to create a robust membrane module, which is important for practical use.

The membranes have good mechanical strength and are capable of withstanding a pressure difference of 4 bars at room temperature. The microfabicated membranes also achieve high permeation rate and high hydrogen selectivity. High flow rates of $3.6 \mathrm{~mol} \mathrm{H}_{2} / \mathrm{m}^{2} . \mathrm{s}$ are measured with minimal selectivity of 1500 for $\mathrm{H}_{2} / \mathrm{He}$.

The reported palladium-silver alloy membranes can be used for hydrogen separation or purification from gas mixtures or membrane reactors for hydrogenation/dehydrogenation reactions.

The results indicate that an industrial pilot module that consists of a stack of a number of glass/membrane plates with a higher throughput of hydrogen becomes feasible.

\section{Acknowledgements}

The financial support from the STW foundation, ABB Lummus Global Inc., DSM and Aquamarijn is kindly acknowledged. Our thanks are also due to the entire staff of $\mathrm{MESA}^{+}$Research Institute, University of Twente, for technical support.

\section{References}

[1] R.Ramachandran et al., Int.J.Hydro. Ener., Vol. 23, 1998, P.593.

[2] B.C.H.Steele et al., Nature 414, 2001, P. 338-344.

[3] United States Department of Energy, "A review of the national hydrogen vision meeting" Washington, DC, November 15-16, 2001.

[4] J.Shu et al., Can.J.Chem.Eng., Vol. 69, 1991, p.1036.

[5] R.Hughes, Memb.Tech. Vol. 131, 2001,P.9.

[6] R.Dittmeyer et al. J.Mol.Cat. A., Vol. 173, 2001, P.135

[7] V.M.Gryaznov, Plat. Met. Rev., Vol. 30, 1986, P.68.

[8] K.F.Jensen, Chem.Eng.Scie, Vol.56, (2001), P.293-303

[9] M. U. Kopp et al., Science Vol. 280, 1046-1048 (1998).

[10] K.F.Jensen, Nature, Vol.393, 1998, p.735-736.

[11] A.V. Berg et al.,(ed) Micro total analysis system 2000,KJuwer Academic.

[12] M.Matlosz et al., (ed) IMRET 5, Springer, 2001.

[13] M.Matlzosz et al., Proc. IMRET 5, P.13-22, Springer, 2001

[14] A.J.Frank et al., Proc. IEEE Conf. MEMS'99, P.382.

[15] H.D.Tong et al., Proc.IEEE Conf. MEMS'02, P.268.

[16] J.G.E.Gardeniers et al., J.Vac.Scie. and Tech.A, Vol.14, P.2879.

[17] S.Kuiper, Ph.D thesis, University of Twente, Aug., 2000.

[18] C.J.M. van Rijn et al., J.Mic.Mec.Syst., Vol. 6,1997,P.48

[19] F.C. Gielens et al., Proc.ICOM. Conf., Jul., 2002. 